\title{
A LOCAL ASYMPTOTIC ANALYSIS OF THE DISCRETE FIRST PAINLEVÉ EQUATION AS THE DISCRETE INDEPENDENT VARIABLE APPROACHES INFINITY
}

\author{
Nalini Joshi \\ This paper is dedicated to Martin David Kruskal \\ on the occasion of his seventieth birthday.
}

\begin{abstract}
The first discrete Painlevé equation (dPI), which appears in a model of quantum gravity, is an integrable nonlinear nonautonomous difference equation which yields the well-known first Painlevé equation (PI) in a continuum limit. The asymptotic study of its solutions as the discrete time-step $n \rightarrow \infty$ is important both for physical application and for checking the accuracy of its role as a numerical discretization of PI. Here we show that the asymptotic analysis carried out by Boutroux (1913) for PI as its independent variable approaches infinity also can be achieved for dPI as its discrete independent variable approaches the same limit.
\end{abstract}

\section{Introduction}

Our aim is to study the equation

$$
y_{n+1}+y_{n}+y_{n-1}=\frac{\alpha n+\beta}{y_{n}}+\gamma
$$

in the limit as $n \rightarrow \infty$. Equation (1) is known as the discrete first Painlevé equation (dPI) because the scaling limit $y_{n}=1+h(-2 w(x)+c / 6), x=\sqrt{h} n-b / 2-c^{2} / 24$, with $\alpha=-2 h^{5 / 2}(1+h a), \beta=-\gamma+3+h^{2} b, \gamma=6+h c$, yields the classical first Painlevé equation (PI)

$$
w^{\prime \prime}=6 w^{2}+x
$$

as $h \rightarrow 0$.

PI is the simplest of six well-known nonlinear second-order ordinary differential equations (ODEs) in the complex plane called the Painlevé equations. Their characteristic property that all movable singularities of all solutions are poles is called the Painlevé property. Painlevé [16], Gambier [7], and Fuchs [9] identified them (under some mild conditions) as the only such equations with the Painlevé property whose general solutions are new transcendental functions.

More recently, it was discovered by Ablowitz, et al. [2] that such equations are closely related to integrable partial differential equations $[3,1]$. There is an arsenal

Received December 30, 1995, revised May 21, 1996.

1991 Mathematics Subject Classification: 39A10, 41A60, 30E15.

Key words and phrases: discrete Painlevé equations, nonlinear discrete asymptotics. 
of rich techniques that exhibit the special character of the Painlevé equations as integrable equations. In particular, Painlevé [17] showed that they can be written in terms of ratios of entire functions, Fuchs [9], and later Jimbo et al. [12] (see also [15]) showed they are isomonodromy conditions for associated linear ODEs, and Joshi and Kruskal [14] showed that their connection problems can be solved directly. Consequently, their solutions play a distinguished role as nonlinear special functions. A natural question to ask is: What are their corresponding discrete versions?

The first insight into this question came from a discrete version of PI derived in a model of quantum gravity $[6,8]$. Subsequently, a tour-de-force by Grammaticos, Ramani, and collaborators (see $[11,18]$ and references in [10]) answered this question. They derived integrable discrete versions of the first to the fifth Painlevé equations and identified a property, called the singularity confinement property, that appears to be a discrete version of the Painlevé property. Isomonodromy problems have been found for these discrete Painlevé equations. They appear, therefore, to be integrable and deserve further study as rare examples of nontrivial integrable systems. An asymptotic description of their possible orbits as $n \rightarrow \infty$ is a natural part of this study.

The limit we study, i.e., $n \rightarrow \infty$, is related to the continuum limit. In such a limit, $n$ is typically multiplied by a small parameter $h^{\mu} \rightarrow 0$, where $\mu=1 / 2$ for dPI to PI, while $n h^{\mu}$ remains $O(1)$. Such a limit can only be achieved if $n$ is itself approaching infinity. In order to understand how the orbits of dPI relate to the solutions of PI in the continuum limit, we need to study dPI in the limit $n \rightarrow \infty$. This is crucial also for deciding whether or not $\mathrm{dPI}$ is an accurate numerical discretization of PI.

It should be noted, however, that the limit $n \rightarrow \infty$ is not equivalent to a continuum limit. Continuum limits of dPI involve scaling limits of $y_{n}$ and the parameters $\alpha, \beta$, $\gamma$, in addition to requiring $n \rightarrow \infty$. Moreover, there exist limits of dPI other than the one described above to PI. For example, an alternative continuum limit to PII (the second Painlevé equation) is also known. Nevertheless, for simplicity, we restrict the comparison of our discrete results to those known for PI.

We show that the asymptotic behaviors admitted by dPI as $n \rightarrow \infty$ are qualitatively extremely similar to those found for PI by Boutroux [5]. Boutroux showed that, in general, the asymptotic behaviors of the (first four) Painleve transcendents near infinity in the plane of the independent variable are given by elliptic functions. He studied the behavior of the transcendents along a line of approximate periods of such elliptic functions, and he showed that within certain sectors near infinity, there also exist special behaviors which are asymptotically free of poles. His deductions were facilitated by a transformation of variables that made the asymptotic behaviors more explicit. For PI, Boutroux's transformation is

$$
w(x)=\sqrt{x} u(z), \quad z=\frac{4}{5} x^{5 / 4},
$$

under which PI becomes

$$
u^{\prime \prime}=6 u^{2}+1-\frac{u^{\prime}}{z}+\frac{4}{25} \frac{u}{z^{2}}
$$

where the primes now denote differentiation with respect to $z$. (See [14] for a deduction of Boutroux transformations based on maximal dominant balances.) It is clear that on a path where $u, u^{\prime}, u^{\prime \prime}$ are bounded, and $u^{\prime \prime}$ is of order unity, the leading-order 
behavior of (2) as $|z| \rightarrow \infty$ is given by

$$
u^{\prime \prime} \approx 6 u^{2}+1,
$$

which is solved by Weierstrass elliptic functions. On the other hand, if $u^{\prime \prime} \ll 1$, as $|z| \rightarrow \infty$, the leading-order behavior is given by

$$
u^{2} \approx-\frac{1}{6}
$$

which gives rise to pole-free behaviors.

There exists a Boutroux transformation of dPI that also facilitates its asymptotic study as $n \rightarrow \infty$. An analysis of possible dominant balances of dPI as $n \rightarrow \infty$ shows that there is only one maximal balance

$$
y_{n+1}+y_{n}+y_{n-1} \approx \frac{\alpha n}{y_{n}}
$$

which is achieved when $y_{n}$ grows like $O(\sqrt{n})$. Hence we are led to

$$
y_{n}=\sqrt{n} u_{n}
$$

which transforms dPI to

$$
\begin{aligned}
u_{n+1}+u_{n}+u_{n-1}=\frac{\alpha}{u_{n}} & +\frac{\gamma}{\sqrt{n}}+\frac{\beta}{n u_{n}} \\
& -\left\{\frac{\sqrt{n+1}-\sqrt{n}}{\sqrt{n}}\right\} u_{n+1}-\left\{\frac{\sqrt{n-1}-\sqrt{n}}{\sqrt{n}}\right\} u_{n-1} .
\end{aligned}
$$

In Section 2, we show that this equation is solved to leading order by elliptic functions generally and by pole-free behaviors less generally.

The elliptic-function-type behaviors are described by an energy-like parameter $E$ and a phase-like parameter $\Phi$ which are constant to leading order but vary slowly as $n \rightarrow \infty$. The evolution of $E$ over many periods of the leading-order elliptic function can be studied via an averaging method. We carry out this study in Section 3.

Although qualitatively we obtain very similar results to those found by Boutroux, we point out here that there are differences. In the special case $\gamma=0$, the main result of Section 3 can be regarded as the discrete analogue of the corresponding result found by Boutroux for PI. However, in the case $\gamma \neq 0$, the slow evolution of $E$ has an additional component. This additional complexity is due to the fact that our asymptotic limit $n \rightarrow \infty$ is not exactly a continuum limit. Moreover, the limit $n \rightarrow \infty$ includes the case where $x=n h^{\mu}$ is finite. Our analysis, therefore, includes regions where Boutroux's asymptotic analysis may not be valid (he assumed $x \rightarrow \infty$ ).

What we demonstrate in this paper is that a comprehensive (formal) local asymptotic description of dPI can be carried out as $n \rightarrow \infty$. Although our results are obtained as $n \rightarrow \infty$, a similar leading-order analysis can be carried out in neighbourhoods of any ordinary point $n=n_{0}$. This also yields elliptic-function-type behaviors and pole-free behaviors. Hence our analysis provides further evidence of the special character of dPI as an integrable equation. Ramani et al. have pointed out that dPI has the singularity confinement property by a perturbation analysis carried out around its singularity at $y=0$. Our asymptotic results verify this pole-like singularity structure of the orbits to leading order by showing that locally in patches near infinity, the orbits are given by meromorphic functions. 


\section{Local Asymptotic Results}

Here we analyse (3) to find the locally valid asymptotic behaviors of dPI in the limit $n \rightarrow \infty$. Our results are qualitatively the same as those found by Boutroux for PI.

Suppose that $u_{n}, u_{n+1}, u_{n-1}$ are all $O(1)$. Then we have a maximal dominant balance of (3) given by

$$
u_{n+1}+u_{n}+u_{n-1} \approx \frac{\alpha}{u_{n}}
$$

Henceforth, we adopt the notation

$$
u_{n+1}=: \bar{u}, \quad u_{n}=u, \quad u_{n-1}=: \underline{u} .
$$

Multiplying (4) by $u \bar{u}$ and $u \underline{u}$, respectively, and subtracting the two results, we get

$$
u \bar{u}^{2}+u^{2} \bar{u}-u^{2} \underline{u}-u \underline{u}^{2} \approx \alpha(\bar{u}-\underline{u}) .
$$

Both sides of this equation are exact differences, so we can integrate (actually sum) to get

$$
u \bar{u}^{2}+u^{2} \bar{u}-\alpha(\bar{u}+u)=: E .
$$

Note that $E$ is constant to leading order as $n \rightarrow \infty$, but varies slowly with $n$. That is, $\bar{E}-E$ is not zero but small (in fact, of order $O(1 / \sqrt{n})$ for $n \gg 1$, see (8) below). We will refer to $E$ as an energy-like integral (or parameter) for dPI. Consider the left side of (5) as a polynomial in $x=u, y=\bar{u}$. The value of $E$ (to leading order) defines level curves of this polynomial which are parametrized by elliptic functions (see [4]).

An alternative way to see that the solution involves elliptic functions is to sum the equation once more. Solve (5) for $\bar{u}$ and write the result as

$$
Q(u, E)=\frac{1}{2 u}\left(\alpha-u^{2} \pm \sqrt{P(u, E)}\right)
$$

where

$$
P(u, E):=\left(u^{2}+\alpha\right)^{2}+4 E u .
$$

Then the result can be written as

$$
\frac{u(\bar{u}-\underline{u})}{\sqrt{P(u, E)}}=1
$$

which upon integration gives

$$
\sum_{k=1}^{n} \frac{u_{k}\left(u_{k+1}-u_{k}\right)}{\sqrt{P\left(u_{k}, E_{k}\right)}}=n-\Phi
$$

where $\Phi$ is a constant. The left side is a discrete analogue (Riemann sum) of an elliptic integral of the first kind.

The leading-order elliptic function has two periods. The periods are (in general) complex numbers because the curve defined by (5) has genus two. In other words, $P$ has four branch points (for generic values of $E$ ), and there exist two linearly independent closed contours that enclose a pair of these. We will denote the two periods (given by the two contours $C_{j}$ ) as $\omega_{j}, j=1,2$. 
In fact, the elliptic function is a discrete sampling of the continuum (Jacobian) elliptic functions. So the definition of $\omega_{j}$ requires slightly more explanation. We regard them as being defined by the elliptic integral

$$
\omega_{j}=\oint_{C_{j}} d u \frac{u}{\sqrt{P(u, E)}}
$$

where the path of integration for each given $E$ is a closed contour (as described above) formed by interpolation through the orbits of $u$ parametrized by the phase $\Phi$.

The elliptic-function-type behavior $u$ of dPI possesses these periods only to leading order. However, we nevertheless define $\omega_{j}(E)$ in the same way and regard them as (implicit) functions of $n$ through their dependence on $E$. We also will call them (loosely) periods.

There exist special values of $E$ for which the elliptic functions degenerate to singly periodic (trigonometric) functions. To find these values, rewrite $P$ as

$$
P(u, E)=(u-\rho)^{2}\left(u^{2}+\sigma u+\tau\right) .
$$

Multiplying out the product on the right and equating coefficients gives

$$
\begin{gathered}
\sigma=2 \rho, \quad \tau-2 \rho \sigma+\rho^{2}=2 \alpha, \\
\rho^{2} \sigma-2 \rho \tau=4 E, \quad \rho^{2} \tau=\alpha^{2} .
\end{gathered}
$$

Solving these shows that

$$
\rho^{2}=\alpha / 3, \quad-\alpha
$$

and

$$
E=-\frac{4}{3} \alpha \sqrt{\alpha / 3}, \quad 0,
$$

respectively. For these values of $E$, the elliptic integrals described above degenerate to yield only a single period for $u$.

Now suppose that $u, \bar{u}, \underline{u}$ are still $O(1)$, but $\bar{u} \approx u, \underline{u} \approx u$. Then the first integral (5), although still defined, describes an algebraic function (to leading order). Since $u$ is constant to this order, where the constant is given by

$$
3 u \approx \frac{\alpha}{u},
$$

we write $u=\sqrt{(\alpha / 3)}+v$, where $v \ll 1$. Then (3) becomes

$$
\begin{aligned}
\bar{v}+4 v+\underline{v}=\frac{\gamma}{\sqrt{n}}(1+v / \sqrt{(\alpha / 3)}) \\
+O\left(1 / n, v \bar{v}, v^{2}, v \underline{v}, \bar{v} / n, \underline{v} / n\right) .
\end{aligned}
$$

We get the asymptotic solution

$$
u \approx \sqrt{(\alpha / 3)}+\frac{\gamma}{6 \sqrt{n}}+O(1 / n)
$$

This solution obviously has an infinite algebraic asymptotic series expansion and, therefore, has no poles for $n \gg 1$. For that reason, we call such solutions pole-free behaviors. 
A straightforward calculation shows that a perturbation of a solution $V$ with such behavior, i.e., $u=V+\hat{v}$, gives

$$
\hat{v} \approx c_{ \pm} \lambda_{ \pm}^{n}
$$

where

$$
\lambda_{ \pm}=-2 \pm \sqrt{3}
$$

and $c_{ \pm}$are arbitrary constants. Obviously, $\left|\lambda_{-}\right|>1$ and $\left|\lambda_{+}\right|<1$. So to get a consistent perturbation, we must have $c_{-}=0$. In other words, we get an exponentially small perturbation, multiplied by $c_{+}$, which is hidden beyond all orders of the algebraic expansion (6) as $n \rightarrow \infty$. Since the free parameter $c_{+}$cannot be identified uniquely, the pole-free behavior (6) cannot describe a solution uniquely.

We chose the solution given by $\lambda_{+}$above under the assumption that $n$ is real and positive. However, in general, dPI could be regarded as a mapping posed along a complex line, i.e., $n$ could be complex. Therefore, the perturbation $\hat{v}$ could be given by either $\left(\lambda_{ \pm}\right)^{n}$ or indeed a combination of both (e.g., if $n$ is pure imaginary). In other words, the behaviors given to the first few orders by (6) suffer from Stokes' phenomenon in the complex $n$-plane.

There is one other dominant balance giving rise to pole-free behaviors. In obtaining $V$ above, we assumed that

$$
u \approx c, \quad \bar{u}+\underline{u} \approx 2 c,
$$

and then found the constant $c$ to be given by $\sqrt{(\alpha / 3)}$. An alternative consistent assumption is

$$
u \approx c, \quad \bar{u}+\underline{u} \approx-2 c .
$$

In this case, we find that

$$
u \approx( \pm 1)^{n} \sqrt{-\alpha}+O(1 / \sqrt{n}) .
$$

Again the solutions with this leading-order behavior suffer from Stokes' phenomenon. However, now the Stokes' lines are orthogonal to those of the pole-free behavior given by $V$. Writing $u=W+\hat{w}$ where $W$ is given to leading order by (7), we get

$$
\hat{w} \approx b_{ \pm}( \pm i)^{n}
$$

where $b_{ \pm}$are arbitrary constants. So along purely real directions in the $n$-plane, the perturbation $\hat{w}$ is approximately oscillatory, whereas along purely imaginary directions, one of $b_{ \pm}$must be zero and $\hat{w}$ is hidden beyond all orders of the divergent expansion for $W$.

Along anti-Stokes' lines, i.e., directions along which the perturbations $\hat{v}$ or $\hat{w}$ are approximately oscillatory, the perturbations are not simply linear combinations of the exponentials found above. As for PI (see [13, 14]), they are more accurately represented by (discrete) Fourier series which yield an expansion composed of increasing powers of such exponentials multiplied by algebraic prefactors. We omit the details here for simplicity.

It can be shown that the elliptic-function-type behaviors and the pole-free behaviors are connected in the space of solutions. The latter can be shown to be degenerate limits of the former, attained when $E \rightarrow-(4 \alpha / 3) \sqrt{\alpha / 3}$ or $E \rightarrow 0$. 
Although the number of pole-free behaviors of PI (or PII) differs from that for dPI (PI has two, PII has three), these results are qualitatively the same asymptotic results as those found by Boutroux.

\section{Averaging}

In this section, we analyse the change of the energy-like parameter $E$ (describing the elliptic-function-type behaviors) over a period $\omega_{j}$ of the leading-order elliptic function by using an averaging method.

$E$ fluctuates with $n$. This is clear from the definition (5) which gives

$$
\begin{gathered}
E-\underline{E}=(\bar{u}-\underline{u})\left\{\frac{\gamma u}{\sqrt{n}}+\frac{\beta}{n}-(\sqrt{(1+1 / n)}-1) \bar{u} u\right. \\
-(\sqrt{(1-1 / n)}-1) u \underline{u}\} .
\end{gathered}
$$

Suppose we are given initial values at some point $\Phi,|\Phi| \gg 1$ :

$$
u(\Phi)=1, \quad u(\Phi+1)=p(\neq 0) .
$$

Then we have

$$
E(\Phi)=p^{2}+p-\alpha(p+1) .
$$

Assume the periods $\omega_{j}$ to be those given at $\Phi$ by this value of $E$.

To carry out an averaging method, we assume that there is a smooth, slowly varying function interpolated between the successive points on the orbit defined by $E$ and $\Phi$ (or the initial values above). We wish to study the slow variation of $E$ from one period of the leading-order elliptic function to the next. Let $u$ be

$$
u=U+s
$$

where $U$ represents the slowly-varying part of $u$ and $s \ll U$ represents its fast fluctuations (as $|n| \rightarrow \infty)$. The initial values are now

$$
\begin{aligned}
U(\Phi) & =1, \quad U(\Phi+1)=p \\
s(\Phi) & =0, \quad s(\Phi+1)=0 .
\end{aligned}
$$

Then (3) gives

$$
\begin{aligned}
\bar{U}+U+\underline{U}= & \frac{\alpha}{U} \\
\bar{s}+s+\underline{s}=\alpha & \left(\frac{1}{U+s}-\frac{1}{U}\right)+\frac{\beta}{n(U+s)}+\frac{\gamma}{\sqrt{n}} \\
& -(\sqrt{1+1 / n}-1)(\bar{U}+\bar{s})-(\sqrt{1-1 / n}-1)(\underline{U}+\underline{s}) .
\end{aligned}
$$

Note that $U$ is an elliptic function (by arguments presented in Section 2).

Consider the first integral given by (5). Expanding the solution $u$ as $U+s$ and keeping only terms of order $s$ or $1 / \sqrt{n}$ gives

$$
\bar{s}\left(2 \bar{U} U+U^{2}-\alpha\right)+s\left(\bar{U}^{2}+2 \bar{U} U-\alpha\right)=\gamma \frac{\bar{U} U}{\sqrt{n}} .
$$


This equation can be integrated once more by using the following observations. The defining equation for $U$ gives

$$
\begin{aligned}
\bar{U} U-U \underline{U} & =\bar{U} U+U(\bar{U}+U-\alpha / U) \\
& =2 \bar{U} U+U^{2}-\alpha, \\
-\overline{\bar{U}} \bar{U}+\bar{U} U & =\bar{U}(\bar{U}+U-\alpha / \bar{U})+\bar{U} U \\
& =\bar{U}^{2}+2 \bar{U} U-\alpha .
\end{aligned}
$$

Letting

$$
F:=\bar{U} U-U \underline{U}
$$

we have

$$
\bar{F}=\overline{\bar{U}} \bar{U}-\bar{U} U
$$

Hence (9) becomes

$$
\bar{s} F-s \bar{F}=\gamma \frac{\bar{U} U}{\sqrt{n}} .
$$

There is an integrating factor for this equation. After division by $\bar{F} F$, it becomes

$$
\overline{\left(\frac{s}{F}\right)}-\frac{s}{F}=\gamma \frac{\bar{U} U}{\bar{F} F \sqrt{n}} .
$$

Hence, we can integrate (actually sum) to get $s$ as

$$
s_{n}=\gamma F_{n} \sum_{k=\Phi}^{n-1} \frac{U_{k+1} U_{k}}{F_{k+1} F_{k} \sqrt{k}}
$$

where the sum is understood to be zero in the case $n=\Phi$.

Now consider (8) for $E$. Note that the first term on the right gives

$$
E(\Phi+\omega)-E(\Phi)=\left.\gamma \frac{\bar{U} U}{\sqrt{n}}\right|_{\Phi} ^{\Phi+\omega}+O(1 / \Phi, s / \sqrt{\Phi})
$$

to leading order. However, the contribution of this term is actually smaller than $O(1 / \sqrt{n})$, because $U$ is periodic and

$$
\frac{1}{\sqrt{\Phi+\omega}}-\frac{1}{\sqrt{\Phi}}=O\left(1 / \Phi^{3 / 2}\right)
$$

To get the nonzero slow change of $E$, therefore, we need to consider terms of size $O(1 / n, s / \sqrt{n})$.

At these orders, we get

$$
\begin{array}{r}
E(\Phi+\omega)-\left.E(\Phi) \approx \gamma \frac{\bar{U} s+U \bar{s}}{\sqrt{n}}\right|_{\Phi} ^{\Phi+\omega}+\left.\frac{\beta(\bar{U}+U)}{n}\right|_{\Phi} ^{\Phi+\omega} \\
-\frac{1}{2 \Phi} \sum_{k=\Phi+1}^{\Phi+\omega} U_{k}\left(U_{k+1}-U_{k-1}\right)^{2}
\end{array}
$$


By periodicity and integration by parts, the second ratio on the right can be shown to be zero to leading order. Hence we have

$$
\begin{aligned}
E(\Phi+\omega)-E(\Phi) \approx & \left.\frac{\gamma^{2}}{\Phi}\left\{U_{n+1} F_{n} \sum_{k=\Phi}^{n-1} \frac{U_{k+1} U_{k}}{F_{k+1} F_{k}}+U_{n} F_{n+1} \sum_{k=\Phi}^{n} \frac{U_{k+1} U_{k}}{F_{k+1} F_{k}}\right\}\right|_{\Phi} ^{\Phi+\omega} \\
& -\frac{1}{2 \Phi} \sum_{\Phi+1}^{\Phi+\omega} U_{k}\left(U_{k+1}-U_{k-1}\right)^{2} \\
\approx & \frac{\gamma^{2}}{\Phi}\left(U_{\Phi+1} F_{\Phi}+U_{\Phi} F_{\Phi+1}\right) \sum_{k=\Phi}^{\Phi+\omega-1} \frac{U_{k+1} U_{k}}{F_{k+1} F_{k}} \\
& -\frac{1}{2 \Phi} \sum_{\Phi+1}^{\Phi+\omega} U_{k}\left(U_{k+1}-U_{k-1}\right)^{2} .
\end{aligned}
$$

To simplify this expression, we use the notation $P(u, E)$ defined in Section 2 . Note that

$$
F=U(\bar{U}-\underline{U})=\sqrt{P(U, E)} .
$$

Therefore, we have

$$
\frac{\bar{U} U}{\bar{F} F}=\frac{\bar{U} U}{\sqrt{P(U, E) P(\bar{U}, \bar{E})}} .
$$

Hence (10) becomes

$$
\begin{aligned}
E(\Phi+\omega)-E(\Phi) \approx & \frac{\gamma^{2}}{\Phi}\left(U_{\Phi+1} F_{\Phi}+U_{\Phi} F_{\Phi+1}\right) \sum_{k=\Phi}^{\Phi+\omega-1} \frac{U_{k+1} U_{k}}{\sqrt{P\left(U_{k+1}, E\right) P\left(U_{k}, E\right)}} \\
& -\frac{1}{2 \Phi} \sum_{\Phi+1}^{\Phi+\omega} \frac{P\left(U_{k}, E\right)}{U_{k}} .
\end{aligned}
$$

In the case $\gamma=0$, this result yields

$$
E(\Phi+\omega)-E(\Phi) \approx-\frac{1}{2 \Phi} \sum_{\Phi+1}^{\Phi+\omega} \frac{P\left(U_{k}, E\right)}{U_{k}} .
$$

This is the discrete analogue of the result found by Boutroux. To recognize this, note that

$$
\frac{P\left(U_{k}, E\right)}{U_{k}}=\left(U_{k+1}-U_{k-1}\right) \sqrt{P\left(U_{k}, E\right)},
$$

and so the sum on the right of (11) is a discrete analogue of the elliptic integral

$$
\tilde{\omega}_{j}=\oint_{C_{j}} d u \sqrt{P(u, E)} .
$$

(Note that the derivative of this integral with respective to $E$ is the previously described elliptic integral $\omega_{j}$ and that in making continuum analogies we have ignored numeric factors.) This is what Boutroux [5, p. 319]) found for the evolution of $E$ (called $D$ in his paper). He showed by using this result that along a chain of increasing points $\Phi_{j}$ defined by $\Phi_{0}=\Phi, E_{0}=E(\Phi), \Phi_{j}=\Phi_{j-1}+\omega\left(E_{j-1}\right), E_{j}=E\left(\Phi_{j}\right), E$ 
must be bounded and approach a value for which the leading order elliptic function degenerates to a trigonometric function.

The generic case $\gamma \neq 0$ differs markedly from this (see the remarks made near the end of Section 1) and will form the subject of future explorations.

Acknowledgements. It is a pleasure to thank the Isaac Newton Institute, where this study was first started, and the Australian Research Council for their support.

\section{References}

1. M. J. Ablowitz and P. A. Clarkson, Solitons, Nonlinear Evolution Equations and Inverse Scattering, Cambridge University Press, Cambridge, 1991.

2. M. J. Ablowitz, A. Ramani, and H. Segur, A connection between nonlinear evolution equations and ordinary differential equations of P-type I and II, J. Math. Phys. 21 (1980), 715-721, 10061015.

3. M. J. Ablowitz and H. Segur, Solitons and the Inverse Scattering Transform, SIAM, Philadelphia, 1981.

4. M. Abramowitz and I. Stegun, Handbook of Mathematical Functions, Dover, New York, 1972.

5. P. Boutroux, Recherches sur les transcendantes de M. Painlevé et l'étude asymptotique des équations différentielles du second ordre, Ann. École Norm. 30 (1913), 265-375.

6. E. Brezin and V. Kazakov, Exactly solvable field theories of closed strings, Phys. Lett. B236 (1990), 144-150.

7. B. Gambier, Acta Math. 33:1 (1910).

8. A. S. Fokas, A. R. Its, and A. V. Kitaev, Discrete Painlevé equations and their appearance in quantum gravity, Comm. Math. Phys. 142 (1991), 313-344.

9. R. Fuchs, Math. Annalen 63 (1907), 301-321.

10. B. Grammaticos and A. Ramani, Discrete Painlevé equations: derivation and properties, In: Applications of Analytic and Geometric Methods to Nonlinear Differential Equations (Ed. P. A. Clarkson), pp. 299-313, 1993.

11. B. Grammaticos, A. Ramani, and V. Papageorgiou, Do integrable mappings have the Painlevé property? Phys. Rev. Lett. 67 (1991), 1825.

12. M. Jimbo, T. Miwa, Y. Mori, and M. Sato, Physica D 23 (1980), 1-80.

13. N. Joshi and M. D. Kruskal, Connection results for the first Painlevé equation, In: Painlevé Transcendents (Eds. D. Levi and P. Winternitz), pp. 61-79, Plenum Press, 1992.

14. _ The Painlevé connection problem: an asymptotic approach I, Stud. Appl. Math. 86 (1992), 315-376.

15. K. Okamoto, Isomonodromic deformation and Painlevé equations and the Garnier system, J. Fac. Sci. Univ. Tokyo Sec. IA Math, 33 (1986), 575-618.

16. P. Painlevé, Acta Math. 25:1 (1902).

17. _ Bull. Soc. Math. 28 (1902), 201-261.

18. A. Ramani, B. Grammaticos, and J. Hietarinta, Discrete versions of the Painlevé equations, Phys. Rev. Lett. 67 (1991), 1829.

School of Mathematics, University of New South Wales, Sydney NSW 2052, Australia Current address: Dept. of Pure Mathematics, University of Adelaide, Adalaide SA5005, Australia

E-mail: njoshi@maths.adelaide.edu.au 